cicatricial tissue, which has caused paralysis of the posterior crico-arytenoid muscle. He would insert a canula, and wait further developments.

Dr. Moure presented a New Nasal Speculu.n. In operating for exploratory puncture of the antrum it is sometimes difficult to withdraw the trocar through the speculum. He has therefore devised a speculum open on the side like a uterine speculum. The ordinary speculum does not open sufficiently at its base, and the author's new instrument is composed of two horizontal cylindrical plates. Good illumination can be obtained, and it can be withdrawn easily. It is meant to replace the speculum of Duplay, commonly used in France.

Dr. Garrigov-Desarènes described his own speculum, which he has used for ten years.

Dr. MIor said that bivalve specula had advantages, and permitted a full view of the interior of the nose during the introduction of instruments.

Dr. Moure said that his new speculum was devised to replace those others, with which we could see perfectly well, but which were inconvenient for certain operations.

\title{
REVIEWS.
}

Maladies de la Bouche et du Phargnx. By Dr. A. Ruault. Paris : Masson, 1892 .

THIs, which forms part of the third volume of the "Traite de Medicine," published under the direction of Drs. Charcot, Bouchard, and Brissaud, is a work such as might have been expected from the reputation of its author. It gives a very complete account of diseases of the mouth and pharynx. The section dealing with diphtheria is especially interesting, comprises no less than 87 pages, is fully up to date, and gives a very complete account of the pathology of this disease.

The author defines diphtheria as a disease caused by a special microorganism, secreting a poison, which, being absorbed, affects the general system. This specific inflammation gives rise to fibrinous pseudomembranes, enclosing the specific bacillus. When situated in the pharynx this is diphtheria; when in the larynx it is croup.

The researches of Klebs, Loeffier, and latterly of Roux and Yersin, are referred to in detail. These latter authors signalized the dependence of the false membranes upon the specific bacillus, the paralyses and other symptoms upon the poison evolved, and the secondary infections upon the micro-organisms which accompany the Klebs bacillus, the streptococcus of Fehleison especially increasing the virulence. A great many researches (Morel, Grancher, Barbier especially) have supplemented those of Roux and Yersin, and suffice to lead to the differentiation of certain clinical forms of the disease, and as indications for prophylaxis. A very complete and carefully written account is given of the etiology and pathogenesis, and precise prophylactic rules are laid down.

Isolation of the patient is not sufficient, but all objects which have 
been in contact with him should be carefully disinfected, which ought to be performed with boiling water or steam. All secretions and even fæcal matters (since false membranes may be swallowed) should be disinfected. Persons coming in contact with the patient should only enter the room when clothed in a blouse or cloak reaching to the feet and covering all their clothing. Disinfection of the hands is of extreme importance. This cannot be done with boiling water, and ordinary antiseptics, including liquor Van Swieten, are not sufficient, since the diphtheritic virus is very resistant to microbicides. Of all antiseptics, phenic acid has been shown in large doses to be the most effective. A solution of phenol in sulphoricinic acid (2o per cent.) is only effective. Cultures remain sterile only when the phenol is to per cent. in glycerine, or 20 per cent. in oil. The sulphoricinic phenol is the only solution which, being antiseptic and non-caustic, is serviceable for disinfection of the hands. The hands should be dry when put into it, and afterwards rinsed clean with a large body of water.

The diverse forms under which diphtheria is manifested may all be reduced to two forms-..

1. Pure diphtheria, monomicrobian or bacillary.

2. Polymicrobian diphtheria, of which there exist two forms, a coccobacillary and a streptococco-bacillary.

The first form is the "normal " of Trousseau, the "toxic" of Grancher, the "diphtheroid" of Lasèue, and the "abortive diphtheria" and "toxic diphtheria" of others.

The false membranes present at their superficies a layer of Klebs bacilli, or along with a few indifferent micro-organisms, generally streptococci of feeble virulence. The onset of the disease is invariably very insidious; the throat affection is very slight, and often absent; the submaxillary glands are swollen. At first the throat is only a little reddened, subsequently false membranes appear (twenty-four to thirtysix hours), generally at first discrete, later continuous. Fever is slight at first, and disappears at the end of two or three days. At the end of eight to twelve days cure of the throat is complete, and general symptoms amend. The false membranes may spread to the nose and neighbouring parts, or larynx (croup), increasing the general intoxication and danger (asphyxia, broncho-pneumonia). It is this form of diphtheria in which local treatment yields the best results.

The second form (polymicrobian) belongs to the kind commonly called "grave, toxic, hypertoxic, malignant and infectious" (Grancher). It is with this that should be classed those secondary diphtherias which occur at the close of certain infectious diseases, e.g., scarlatina, measles, whooping-cough, typhoid, etc.; also should be included cocco-bacillary diphtheria (Barbier), since it differs from streptococcal severe diphtheria. Its characters are glandular tumefaction without puffiness, false membranes, mixed with muco-purulent secretions, resting on a red and tumefied mucous membrane, and muco-purulent expectoration. Certain other forms may on further study be included with this type. These are not accompanied with considerable glandular tumefaction, at least not 
with puffiness, and do not cause very marked anæmia and prostration. The appetite is preserved better than in true diphtheria, and albuminuria is slight; but the false membrane is thick, stratified, greenish, rapidly invading; the mucous membrane below is red, sometimes bleeding at points, and expectoration is slight or moderate, and the breath devoid of fœetidity. The local condition would recall malignant diphtheria. Evolution is variable, and the patient is exposed to all the toxic and infectious complications of the infectious strepto-bacillary form. Most of these cases are modifications of the latter, since, in patients who have succumbed to secondary broncho-pulmonary or croupous complications, without septicamia, the cervical glands and lungs are found to be invaded by the streptococcus.

The streptococcal-bacillary form may appear in the course of purely diphtheritic angina. It then occurs during its first period-during its defervescence-or even when nearly completely cured, and the signs and symptoms appear only secondarily. It may also occur during the course or at the end of a simple angina, caused by the streptococcus, or it may appear suddenly; the debut is almost always sudden and violent. Fever varies in intensity: there may be rigors and elevation of temperature, or the latter may be temporary or insignificant, or even hypothermic. Its suddenness is evidenced by the rapidity with which the general toxic and septicæmic symptoms are accentuated (anamia, prostration), and the suddenness of the local symptoms, the mucosa being the seat of tumefaction as soon as it becomes red, sanious, or bleeding, and covered with thick, soft, putrid, brown or yellow false membranes, exfoliating and reforming with rapidity, and horribly foetid. From the commencement the submaxillary and sterno-mastoid glands swell, and this becomes considerable. Diffuse œdematous infiltration of the cellular tissue occurs, with which the glands are confused.

Pain of the throat is very early, and soon becomes intense, the general condition aggravates, adynamia becomes more pronounced, and the patient less sensible. The face swells, and becomes cyanosed. The urine often contains a great quantity of albumen. Diarrhœa often occurs. The false membranes spread to the nose, middle ear, and conjunctiva, occasioning sero-fibrinous or sanguinolent secretion, or completely hæmorrhagic. Invasion of the larynx and respiratory passages is very frequent.

In the gravest cases evolution is so rapid that the patient succumbs to intoxication and septicæmia before the pseudo-membranes have time to extend or any infectious complication to be produced. Prostration is great, increased so that in three or even two days, or even twenty-four hours, death occurs from collapse or syncope.

If the course is slower, complications of croup and broncho-pneumonia occur, or toxic accidents, like paralysis, endocarditis, etc.

In favourable cases the general condition sometimes begins to amend at the same time as the throat. A patient may escape the severer sequela, or be affected only with curable complications (paralyses, glandular suppurations, otitis, etc.), and may after long and slow convalescence be cured definitely. In all cases he will be left with alterations of the throat, 
losses of suostance of the tonsils or palate, or with chronic pharyngitis. In young subjects persistent glandular swellings are frequent.

It will be seen that $M$. Ruault endeavours to classify the different types of diplitheria upon a very scientific basis. The importance of bacteriology is nowhere more markedly exhibited than in the results which have ensued from its application to the study of this disease. We may say that we are now beginning to fairly comprehend a disease which has long baffled physicians, and to this end no scientists have contributed more solidly than our French confrères.

It scarcely needs to be said that $M$. Ruault places great reliance on the bacteriological diagnosis of the disease. It, however, takes twenty to twenty-four hours for the inoculated tubes of serum to develop the characteristic colonies, and occasionally three or four days. The microscopic test of Roux and Yersin, at first simple spreading of the secretion on cover glasses, then sections, permits, if the results are negative for several days, of the exclusion of diphtheria. If, however, bacilli are very numerous, the onset of diphtheria is very probable. An ideal test-which, however, is yet wanting-would be a chemical reaction given by the diphtheritic poison in the urine, or vatery extract of the false membranes. Treatment, as necessarily follows from the author's exposition of the disease, must be severely local--to remove and destroy the false membranes-and general, to control the poison in the system. For the former nothing equals phenic acid, swabbed and irrigated, and for the latter quinine and antiseptics like benzoate of naphthol.

In the chapter upon hypertrophy of the tonsils, the author counsels that in children who have hypertrophy of the pharyngeal tonsil along with soft enlarged patatine tonsils, the former should first be removed, the palatine tonsils frequently rapidly diminishing in size after the post-nasal operation. He speaks favourably of ignipuncture, especially with the modified Paquelin cautery.

The acute non-specific anginas are defined by Ruault as common acute inflammations developing under the infiuence of phlogogenous micro-organisms, devoid of specificity. As these phlogogenous agents do not possess any distinctive clinical or anatomical characters, the classification of these disorders cannot be etiological.

They are thus different degrees of inflammation modified by the site; i.e., superficial or deep. Amongst the micro-organisms which occur in these inflammations are those which are commonly found in the mouth, especially staphylococcus albus, streptococcus pyogenes, and Talamon's pneumococcus, and it is to this latter that secondary lesions more or less distant from the throat are chiefly due. Why these organisms, which normally exist in the mouth, do not cause pathological conditions, is due to phagocytosis. When the ordinary conditions of the struggle between. the mucous membrane and the micro-organisms are modified, the latter increase, and through the failure of resistance of the former find a means of entry. This question of phagocytosis is discussed fully by the author. It is both the quantity and the quality of the micro-organisms which will determine the nature of the inflammation resulting. 
Inflammation is a defensive act of the mucous membrane, and its intensity will depend upon the energy exhibited by the organism to attain the destruction of the infective agents, and the time necessary to accomplish it. If these agents reach the sub-mucous layer, the reaction of this will cause a phlegmonous inflammation, terminating in suppuration when the leucocytes succumb in too great number. Under the influence of many conditions destruction of the tissues will become greater, and a sphacelus or gangrene will result, with general intoxication. The microbes may not all be destroyed in situ, but some may penetrate the circulation, arriving at distant places, where resistance is slight from some old trouble. Just as a primary angina may lead to general infection, the latter may give rise to a secondary angina of which Kannenberg, Landouzy, Dubousquet-Laborderie, and Joal have given examples. The cases recorded by Froelich are typical.

We have not space to follow the author further in these interesting studies. We have cited enough to show that M. Ruault has contributed a volume which is imbued with the scientific spirit, and which is ance a learned and a practical contribution to the literature of diseases of the pharynx. In this volume the old lines of book-making are abandoned, and a serious and, so far as it can be, successful effort is made to supply a basis of bacterio-pathology to the subject. The result is a work which no physician can afford to ignore.

R. Norris Wolfenden.

\section{Transactions of the Nineteenth Annual Meeting of the American Laryngological Association. New York: Appleton \& Co., 1892.}

THIS volume, which contains seventeen papers, published in full, as presented by various members of the Association at the annual meeting held in Washington in 1891, bears ample testimony to the high scientific standard of the work done by this Association, which is the doyen of all similar associations, and must always, so long as it maintains its present high standard, be the model of what similar societies should endeavour to attain to. This particular volume bears a melancholy interest from the fact that therein is printed, as an appendix, the inaugural thesis of the late Dr. Frank H. Potter, entitled "Cystoma of the Nasal Passages, with Report of a Case," and this is followed by a sympathetic obituary notice of this talented young laryngologist. It is unnecessary to refer in detail to the work recorded in this volume, since all has received notice previously in this Journal. We may be allowed to congratulate the Association upon its flourishing condition.

R. Norris Wolfenden.

Annual of the Universal Medical Sciences. Edited by Dr. SAjous. Philadelphia : F. A. Davis, 1892.

TH1s colossal work is well known, and that it has reached its fifth year of publication, with no falling off from the standard of excellence originally attained, is a tribute to the untiring and immense zeal of Dr. Sajous and his army of co-cditors and collaborators, and to the great enterprise of the publishers. It is certainly one of the most magnificent scientific 